\title{
Article \\ Molecular Landscape of the Coagulome of Oral Squamous Cell Carcinoma
}

\author{
Marine Lottin ${ }^{1,2} \mathbb{D}^{D}$, Simon Soudet ${ }^{1,3} \mathbb{D}^{D}$, Julie Fercot ${ }^{1,2}$, Floriane Racine ${ }^{1,2} \mathbb{D}$, Julien Demagny ${ }^{4}$, Jérémie Bettoni ${ }^{1,5}$, \\ Denis Chatelain ${ }^{1,6}$, Marie-Antoinette Sevestre ${ }^{1,3}$, Youcef Mammeri ${ }^{7}$ (D), Michele Lamuraglia ${ }^{8}$, \\ Antoine Galmiche ${ }^{1,2, *(D)}$ and Zuzana Saidak ${ }^{1,2, *}$
}

1 EA7516 CHIMERE, Université de Picardie Jules Verne, 80054 Amiens, France; Lottin.Marine@chu-amiens.fr (M.L.); Soudet.Simon@chu-amiens.fr (S.S.); julie.fercot@etud.u-picardie.fr (J.F.); floriane.racine@etud.u-picardie.fr (F.R.); Bettoni.Jeremie@chu-amiens.fr (J.B.); Chatelain.Denis@chu-amiens.fr (D.C.); sevestre.marie-antoinette@chu-amiens.fr (M.-A.S.)

2 Department of Biochemistry, Center for Human Biology, Amiens University Hospital, 80054 Amiens, France

3 Department of Vascular Medecine, Amiens University Hospital, 80054 Amiens, France

4 Department of Hematology, Center for Human Biology, Amiens University Hospital, 80054 Amiens, France; Demagny.Julien@chu-amiens.fr

5 Department of Maxillofacial Surgery, Amiens University Hospital, 80054 Amiens, France

6 Department of Pathology, Amiens University Hospital, 80054 Amiens, France

7 Laboratoire Amiénois de Mathématique Fondamentale et Appliquée (LAMFA), CNRS UMR7352, Université de Picardie Jules Verne, 80069 Amiens, France; youcef.mammeri@u-picardie.fr

8 Department of Oncology, Amiens University Hospital, 80054 Amiens, France; Lamuraglia.Michele@chu-amiens.fr

* Correspondence: galmiche.antoine@chu-amiens.fr (A.G.); saidak.zuzana@chu-amiens.fr (Z.S.)

\section{check for} updates

Citation: Lottin, M.; Soudet, S.; Fercot, J.; Racine, F.; Demagny, J.; Bettoni, J.; Chatelain, D.; Sevestre, M.-A.; Mammeri, Y.; Lamuraglia, M.; et al. Molecular Landscape of the Coagulome of Oral Squamous Cell Carcinoma. Cancers 2022, 14, 460. https://doi.org/ $10.3390 /$ cancers 14020460

Academic Editors: Marilena Vered and Lorenzo Lo Muzio

Received: 17 December 2021

Accepted: 15 January 2022

Published: 17 January 2022

Publisher's Note: MDPI stays neutral with regard to jurisdictional claims in published maps and institutional affiliations.

Copyright: (c) 2022 by the authors. Licensee MDPI, Basel, Switzerland. This article is an open access article distributed under the terms and conditions of the Creative Commons Attribution (CC BY) license (https:// creativecommons.org/licenses/by/ $4.0 /)$.
Simple Summary: Cancer is associated with a wide spectrum of hemostatic complications that range from thrombotic events to hemorrhage. The tumor coagulomes, i.e., the essential actors that locally regulate coagulation and fibrinolysis, play a key role in these complications. They might also play a regulatory role in various cell types of the tumor microenvironment. Here, we explored the coagulome of Oral Squamous Cell Carcinoma (OSCC) across tumor types, between OSCC tumors and within individual tumors. The coagulome of OSCC is characterized by a high expression of antipodal activators of coagulation and fibrinolysis, and subpopulations of pro-coagulant and pro-fibrinolytic cancer cells coexist within individual tumors. Importantly, we noted that dendritic cells within OSCC with a procoagulant profile express high levels of key immune checkpoint molecules. Further studies examining a possible negative modulation of the tumor's adaptive immune response by the coagulation process are warranted.

\begin{abstract}
Background: Hemostatic complications, ranging from thromboembolism to bleeding, are a significant source of morbidity and mortality in cancer patients. The tumor coagulome represents the multiple genes and proteins that locally contribute to the equilibrium between coagulation and fibrinolysis. We aimed to study the coagulome of Oral Squamous Cell Carcinoma (OSCC) and examine its link to the tumor microenvironment (TME). Methods: We used data from bulk tumor DNA/RNA-seq (The Cancer Genome Atlas), single-cell RNA-seq data and OSCC cells in culture. Results: Among all tumor types, OSCC was identified as the tumor with the highest mRNA expression levels of F3 (Tissue Factor, TF) and PLAU (urokinase type-plasminogen activator, uPA). Great inter- and intra-tumor heterogeneity were observed. Single-cell analyses showed the coexistence of subpopulations of pro-coagulant and pro-fibrinolytic cancer cells within individual tumors. Interestingly, OSCC with high F3 expressed higher levels of the key immune checkpoint molecules CD274/PD-L1, PDCD1LG2/PD-L2 and CD80, especially in tumor dendritic cells. In vitro studies confirmed the particularity of the OSCC coagulome and suggested that thrombin exerts indirect effects on OSCC cells. Conclusions: OSCC presents a specific coagulome. Further studies examining a possible negative modulation of the tumor's adaptive immune response by the coagulation process are warranted.
\end{abstract}


Keywords: tumor coagulome; OSCC; tumor microenvironment; immune checkpoints

\section{Introduction}

A broad spectrum of hemostatic complications, ranging from thromboembolism to hemorrhage, represent a significant source of morbidity and mortality in cancer patients $[1,2]$. Oral squamous cell carcinoma (OSCC) represents the most frequent histological form of tumor of the oral cavity and arises in the context of chronic exposure to tobacco and alcohol [3]. Patients with OSCC receive surgical procedures and medical treatments that put them at risk of thrombosis and hemorrhage [4-6], but they are usually considered to be at low risk of systemic thrombosis $[7,8]$. Understanding the biological basis for patient susceptibility to this large spectrum of complications requires fundamental studies on tumor physiology.

The concept of the tumor coagulome refers to the equilibrium that exists between coagulation and fibrinolysis within tumors, and the contribution of multiple genes and proteins to this equilibrium [9]. Over the past decade, systems biology approaches, especially using genomics, have been applied to study the "core» components of the tumor coagulome, i.e., the local regulation of the main effectors of coagulation [10-13]. Clot formation is induced by the polymerization of fibrin resulting from thrombin activity, and it is initiated by the Tissue Factor (TF) encoded by the gene F3 [14]. The overexpression of F3 is a pivotal event in the establishment of the hypercoagulable state that characterizes most human tumors [14]. Conversely, fibrinolysis, the process that leads to clot destruction, depends on the activation of plasmin, involving the plasminogen activators uPA and tPA encoded by the genes PLAU and PLAT, respectively. In addition to the regulation of clot formation, the protease thrombin can also have a direct effect on cancer cells and the cells of the tumor microenvironment (TME) through its ability to interact with the protease-activated receptor-1 (PAR-1), a G-protein coupled receptor encoded by the gene F2R. The interaction of thrombin with PAR-1 modulates the growth of cancer cells and exerts complex effects on the malignant phenotype [15]. The serine protease uPA interacts with the receptor uPAR (urokinase-type plasminogen activator receptor, encoded by the gene PLAUR). Together with the serpin inhibitor plasminogen activator inhibitor-1 (PAI-1, encoded by the gene SERPINE1), uPA and uPAR are well-established markers of tumor prognosis [16].

Compared to other primary tumors, Head and Neck Squamous Cell Carcinoma (HNSCC), including OSCC, express high levels of pro-coagulant (F3) and pro-fibrinolytic $(P L A U)$ genes [13]. The co-expression of high levels of pro-coagulant and pro-fibrinolytic genes establishes OSCC as an interesting model. Why OSCC may have such a specific coagulome is presently unclear as other sublocations of HNSCC, such as oropharyngeal tumors that arise in the context of chronic infection with oncogenic Human Papillomavirus (HPV), are characterized by low mRNA levels of F3 and PLAU [17]. Interestingly, the oral cavity represents a specific biological environment with unique properties, such as a remarkable healing ability [18]. Coagulation contributes to wound healing and to the installation of the phenomenon of a «wound that does not heal» that characterizes most tumors [19]. With this in mind, a systems-level exploration of the coagulome of OSCC is interesting from both a medical and a fundamental perspective.

The tumor microenvironment (TME) represents the non-malignant cells and structures (e.g., extracellular matrix) that are found within tumors $[20,21]$. The various cellular components of the TME, including cancer-associated fibroblasts, vascular cells and immune cells of myeloid or lymphoid lineages, can play a pro- or anti-tumor role depending on the context $[20,21]$. The TME is not only increasingly recognized as a critical determinant of tumor progression but is also emerging as a therapeutic target, as recently shown by the advent of immune checkpoint blockers [22]. A key checkpoint of the adaptive immune response against tumors is the interaction between the programmed cell death protein-1 (PD-1) molecule and its ligands PD-L1 and PD-L2 encoded by the CD274 and PDCD1LG2 genes, respectively. The expression of PD-L1 or PD-L2 on the surface of cancer cells and 
antigen-presenting cells induces a state of anergy in T cells. Targeting this interaction with clinically approved molecules was shown to be efficacious against recurrent/metastatic stages of HNSCC in phase 3 trials [22]. A second important immune checkpoint that can be targeted for therapeutic purposes depends on the interaction between Cytotoxic TLymphocyte Associated Protein 4 (CTLA4) and its ligand CD80. Understanding in which context these checkpoints are involved is an important research objective in order to optimize the efficacy of immune checkpoint inhibitors (ICI) and identify biomarkers for therapeutic stratification [23].

Importantly, a number of studies suggest the existence of an interplay between the coagulation system and the TME. The coagulation system has a potent effect on myeloid cells and initiates inflammation during the physiological healing process [24]. In the TME, coagulation might contribute to the recruitment of cells of the myeloid/lymphoid lineages and modulate their activation and function [25,26]. In the present study, we aimed to explore the regulation of the coagulome of OSCC and its link to the TME of these tumors.

\section{Materials and Methods}

\subsection{Bulk RNA-Seq and DNA Methylation Analysis from Human Tumors}

RNA-seq is a powerful method for transcriptomic analyses. The results of bulk RNA sequencing of tumors can be obtained from a number of freely available resources, such as The Cancer Genome Atlas (TCGA). In this study, we retrieved gene expression and methylation data from TCGA [27] using cbioportal: https: / / cbioportal.org (accessed on 1 October 2021). Gene expression was given as RSEM (RNA-Seq by Expectation-Maximization), an algorithm used to estimate the gene expression based on the number of sequencing reads, after correcting for potential bias. HumanMethylation 450 is an array-based method that permits the quantification of methylation of $450,000 \mathrm{CpG}$ dinucleotide $(96 \%$ of CpG islands and $92 \%$ of $\mathrm{CpG}$ shores are represented by at least one probe). HM450 beta values were retrieved from cBioPortal and used for the analysis ( $0=$ unmethylated, $1=$ full methylated $)$. The data on gene mutations and copy-number alterations were retrieved from cBioPortal (TCGA cohort) for the genes F3, PLAU, PLAUR, PLAT, SERPINE1 and F2R. The clinical characteristics of the 321 OSCC tumors available in TCGA are summarized in Table S1.

\subsection{Single-Cell Transcriptomics}

Compared to tumor bulk RNA-sequencing, single-cell RNA-sequencing (scRNA-seq) permits cell type-specific analyses and provides access to intra-tumor heterogeneity. Singlecell RNA-seq data from 5902 cells were obtained from GSE103322 [28]. Pre-processing and quality control of the scRNA-seq data are described in detail in Puram et al. [28]. The data include 2215 malignant cells obtained from $18 \mathrm{HPV}^{-v e}$ OSCC. The data on a sufficiently high number of cancer cells were available for further analysis for 10 of these tumors.

\subsection{Tumor Microenvironment Analysis}

The ESTIMATE method uses gene expression signatures to infer the fraction of stromal (i.e., fibroblastic/mesenchymal cells) and immune cells in tumor samples and is described in [29]. The ESTIMATE score is based on two gene signatures ('immune signature' and 'stromal signature'). The genes associated with the immune signature were identified using leukocyte methylation scores, shown to correlate with the presence of leukocytes. The genes present in the stromal signature were selected by comparing non-hematopoiesis genes in the tumor cell fraction and matched the stromal cell fraction in different cancer data sets. More detail about the construction of these gene scores can be found in Yoshihara et al. [29].

\subsection{Gene Set Enrichment Analysis (GSEA)}

GSEA, a computational method used to determine whether a set of genes shows statistically significant, concordant differences between two biological states, was performed using the Java GSEA desktop application (https:/ / www.gsea-msigdb.org/gsea/index.jsp, accessed on 1 October 2021). We used curated hallmark gene sets, downloaded from the 
GSEA website, to compute their over-representation in RNA-seq samples expressing F3 vs. PLAU in cancer cells from tumor \#20 from GSE103322. The analyses were conducted using 1000 permutations [30].

\subsection{Cell Culture and Reagents}

The human OSCC cell lines PECA/PJ34, PECA/PJ41 and SCC9 were purchased from LGC Standards (Strasbourg, France) and compared to three human glioma cell lines (A172, U118MG and SW1088). Cell lines were authenticated using short tandem repeat profiling (LGC Standards, Strasbourg, France) and cell cultures were routinely checked for mycoplasma contamination. All cell lines were cultured in Dulbecco Modified Eagle's Medium (DMEM, purchased from Sigma Aldrich (Saint-Quentin Falavier, France) supplemented with 5\% fetal calf serum, 1\% added glutamine and antibiotics (all from Sigma Aldrich). Thrombin and dabigatran were purchased from Sigma Aldrich. Human Interferon $-\gamma$ was purchased from R\&D Systems (Minneapolis, MI, USA). The growth of cancer cells was monitored after one rinse with PBS, a step of fixation with methanol and staining with crystal violet (Sigma Aldrich, Saint Quentin Falavier, France). After a wash step, the crystal violet dye was solubilized and measured by absorbance at $570 \mathrm{~nm}$. The results were expressed as \% of control.

\subsection{Antibodies and Immunoblot Analysis}

The procedures used for immunoblot analysis are reported in detail elsewhere [31]. The primary antibodies used in this study were: anti-TF (TF9-10H10, Sigma Aldrich), antiuPA (Ab169754, Abcam, Paris, France), anti-tPA (Ab157469, Abcam), anti-PAI-1 (Ab66705, Abcam), anti-PAR-1 (E9J9L Rabbit mAb, \#79109, Cell Signaling Technology, Danvers, MA, USA), anti-PD-L1 (Ab213524, Abcam) and anti- $\beta$-actin (A5441, Sigma-Aldrich). For the original Western blots, see Supplementary Materials.

\subsection{Statistical Analyses}

Comparisons of two groups of numeric data were performed using the unpaired Wilcoxon-Mann-Whitney test. In all analyses, the Bonferroni correction was applied to control for multiple testing. Kaplan Meier analyses and the log-rank test were performed to compare the overall survival (OS) and disease-free survival (DFS) between OSCC tumors with high F3 (>median) vs. low F3 expression (<median), high PLAU (>median) vs. low PLAU expression (<median) and high PLAT (>median) vs. low PLAT expression ( $<$ median). $p<0.05$ was set as the threshold for significance. All statistical analyses were performed with R version 4.1.0 (https: / / www.r-project.org, accessed on 1 November 2021). Correlation analyses were carried out using packages Hmisc and corrplot, calculating Spearman correlation coefficients $(\mathrm{r})$.

\section{Results}

\subsection{OSCC Express the Highest Levels of F3/PLAU across Human Tumors}

We examined the mRNA levels of six genes that constitute the "core» tumor coagulome: F3, PLAU, PLAT, PLAUR, F2R and SERPINE1 encoding TF, uPA, tPA, uPAR, PAR-1 and PAI-1, respectively, in tumors from TCGA (Figure 1). A pan-cancer comparison revealed great differences among the different types of primary tumors. Strikingly, OSCC was the human tumor type with the highest expression levels of F3 and PLAU (Figure 1). However, great heterogeneity in F3/PLAU expression was apparent across individual OSCC tumors. The high expression of F3 and PLAU and the tumor heterogeneity were evident from the direct comparison of OSCC and Glioblastoma multiforme (GBM), a tumor type associated with one of the highest risks of thromboembolic events (Figure 2). 
F3

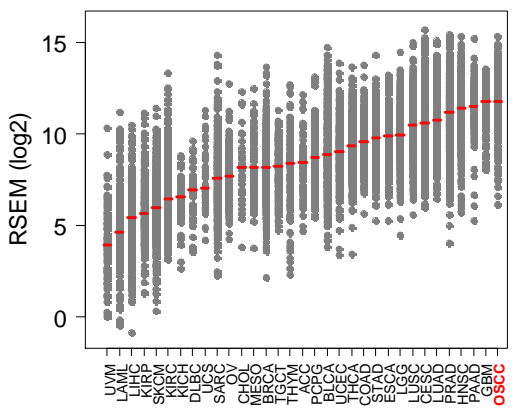

PLAUR

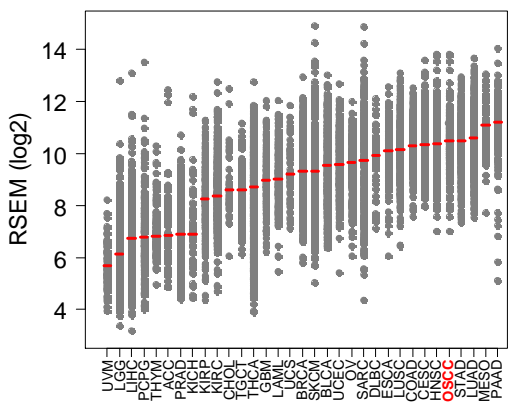

PLAU

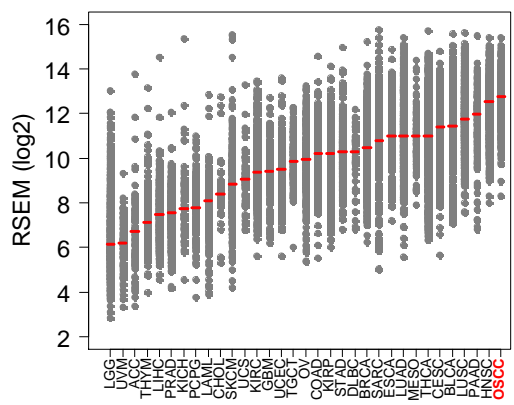

F2R

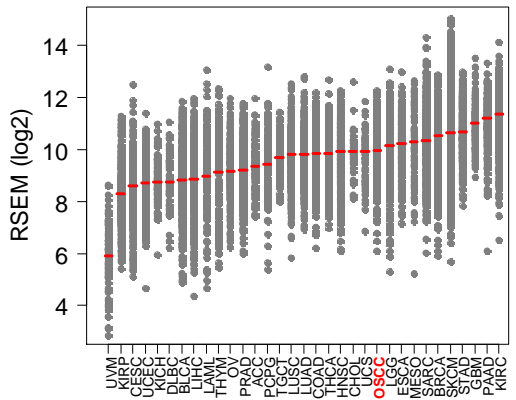

PLAT

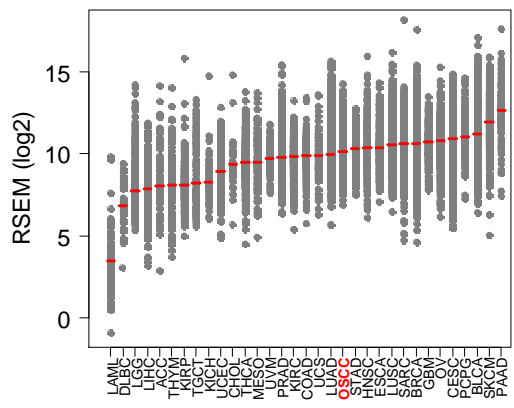

SERPINE1

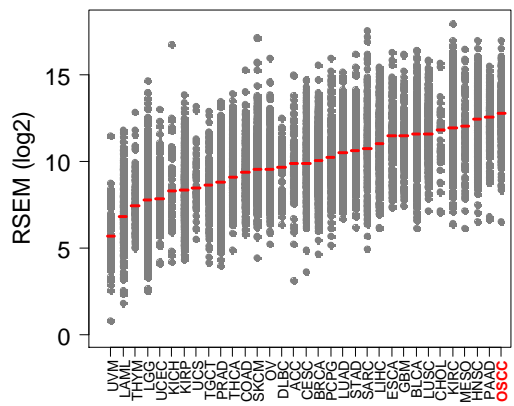

Figure 1. Coagulome gene expression in human tumors. Dot plots showing the tumor type ranking according to the mRNA expression levels of six essential components of the tumor coagulome (F3, PLAU, PLAT, PLAUR, F2R and SERPINE1). Data from $n=33$ tumor types, including OSCC, were retrieved from TCGA (total number of tumors analyzed was $n=10,071$ ). Note that OSCC is the tumor type that presents the highest expression levels of F3, PLAU and SERPINE1 (OSCC in red).

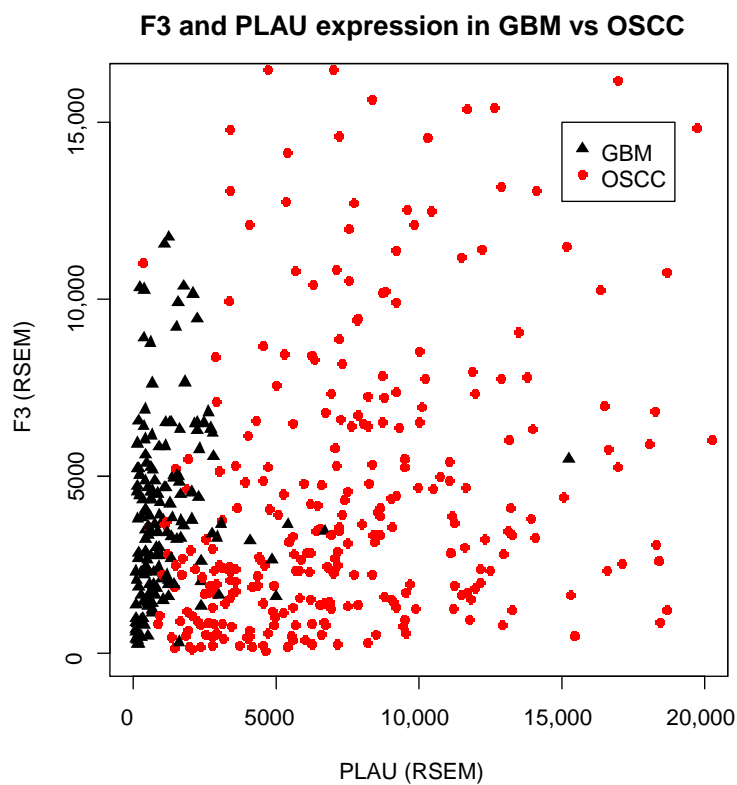

Figure 2. Inter-tumor heterogeneity of $F 3$ and $P L A U$ expression in OSCC and GBM. Comparison of F3 and PLAU expression in OSCC (red, $n=321$ ) and GBM (black, $n=166$ ) from TCGA. mRNA levels are shown as RSEM, showing the great inter-tumor heterogeneity of OSCC tumors. Note that each dot represents an individual tumor.

We examined the extent to which the coagulome of OSCC is correlated with tumor staging (TNM status) and histological grade (Figure S1). We found no significant differences in F3, PLAU, PLAT mRNA expressions according to the $\mathrm{T}$ or $\mathrm{N}$ status. A small yet 
statistically significant difference was noted in PLAU mRNA expression depending on tumor grade, with higher PLAU levels in less differentiated tumors (G2/3) (Figure S1). Tobacco history and patient age were also considered as potentially interesting variables linked to the coagulome of OSCC, but no significant association was found between the expression of the coagulome genes and patient age or number of pack years (Figure S2). Further examination of F3/PLAU expression did not show any significant link to overall survival or disease-free survival of OSCC patients, suggesting that the coagulome is not strongly related to OSCC progression (Figure S3).

\subsection{The Coagulome of OSCC Is Genetically Stable and Is Correlated with Locus-Specific DNA Methylation}

To address the mechanisms that regulate the coagulome, we searched for gene amplifications/deletions/mutations involving the genes of the coagulome in TCGA. The search identified the gene amplifications of PLAT and SERPINE1 as the most frequent genomic events in OSCC, albeit at a low frequency of $2.2 \%$ (Table S2). Somatic tumor mutations in STK11, KRAS, CTNNB1, KEAP1, CDKN2B and MET, which were reported to be associated with cancer-induced venous thromboembolism in an independent study [12], were infrequent in OSCC. We found no statistically significant association of any of the corresponding mutations with the expression of the coagulome in TCGA (data not shown). Meanwhile, there was great variation in locus-specific DNA methylation across tumors: a strong negative correlation (Spearman $\mathrm{r}=-0.65, p=7.72 \times 10^{-40}$ ) was found between F3 mRNA expression and F3 gene methylation. A slightly less strong yet clear negative correlation between mRNA expression and gene methylation was also observed for PLAU and PLAT $\left(\mathrm{r}=-0.44, p=1.93 \times 10^{-16}\right.$ and $\mathrm{r}=-0.42, p=7.85 \times 10^{-15}$, respectively) (Figure 3$)$. We concluded that the coagulome of OSCC is overall genetically stable and that its expression is correlated with locus-specific DNA methylation.
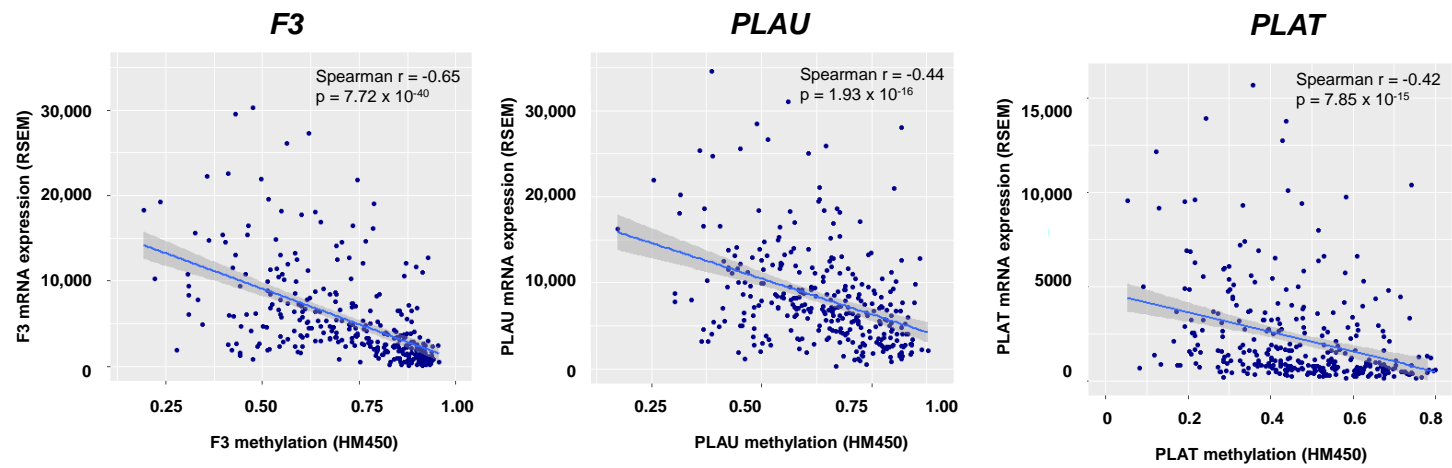

PLAUR

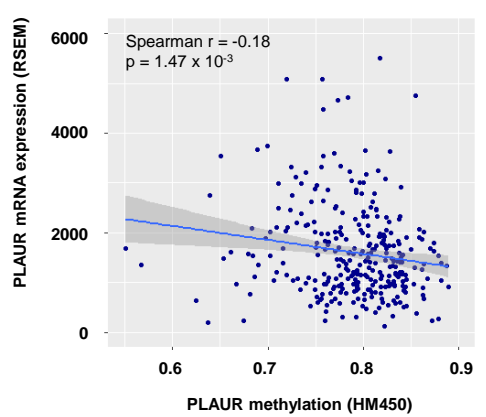

F2R

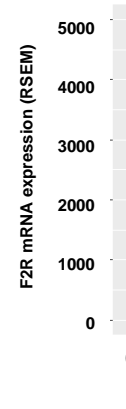

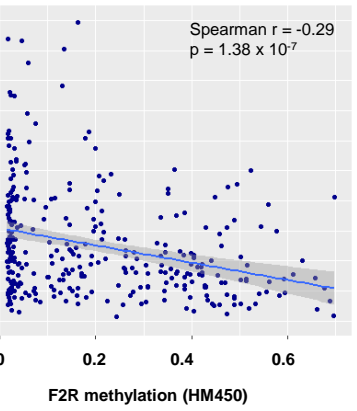

\section{SERPINE1}

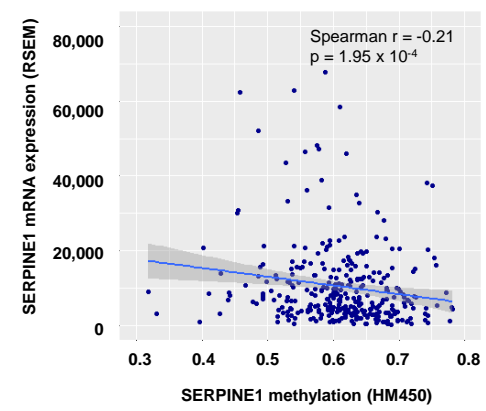

Figure 3. Link between coagulome gene expression and gene methylation. Local DNA methylation was negatively correlated with F3, PLAU and PLAT gene expression. Graphs showing F3, PLAU and PLAT mRNA expressions (RSEM) and the CpG methylation levels of the gene promoter (HM450 beta values, $0=$ unmethylated, $1=$ full methylated $)$. Correlations were performed using Spearman analysis. 


\subsection{The Coagulome of OSCC at the Single-Cell Resolution}

Bulk RNA-seq data reflect gene expression in all cell types, including tumor cells and cells of the TME. We used scRNA-seq data from Puram et al. [28] to analyze the relative expression of each actor of the coagulome in different cell types, as shown in Figures 4 and 5. In OSCC, on average, cancer cells expressed high mRNA levels of F3, PLAU and PLAT, but little if any of PLAUR, F2R or SERPINE1. Conversely, tumor-associated macrophages (TAM) expressed high levels of PLAU and PLAUR, and tumor-associated endothelial cells expressed high levels of PLAT, F2R and SERPINE1 (Figures 4 and 5). Of note, cancer cells were the major contributor to $F 3$ expression in OSCC. Dendritic cells and T cells expressed little, if any, of the core actors of the coagulome (Figures 4 and 5).

F3

PLAU

PLAT
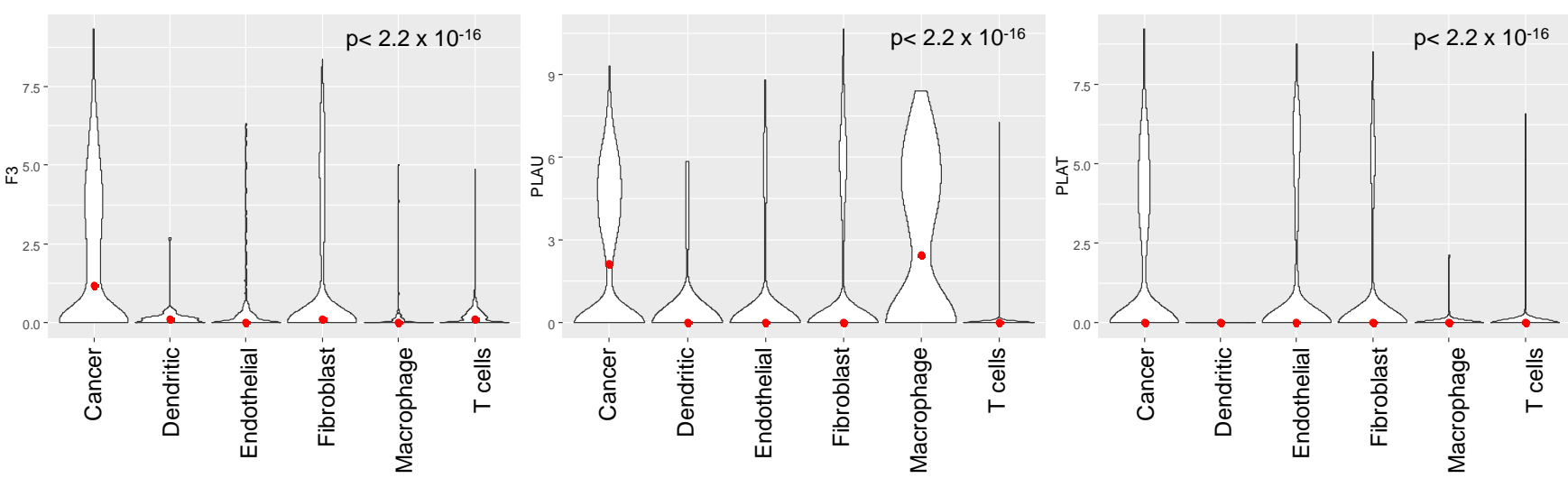

PLAUR

F2R
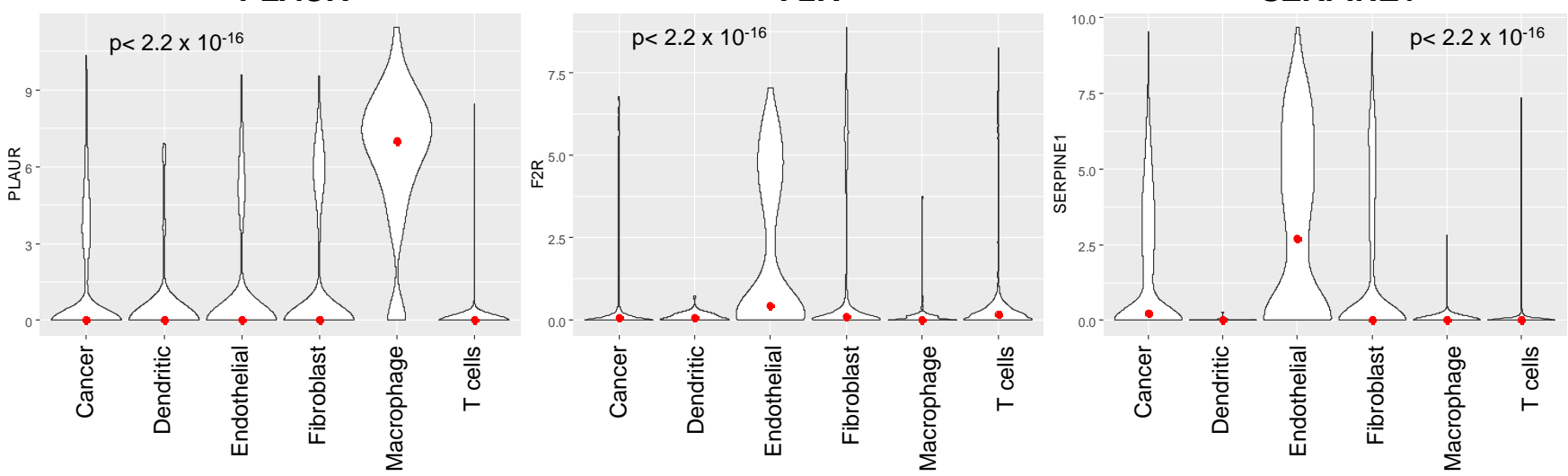

Figure 4. Single-cell analysis of the coagulome in different cell subpopulations in OSCC. Violin plots showing the mRNA expression of the core coagulome genes (F3, PLAU, PLAT, PLAUR, F2R and $S E R P I N E 1$ ) in different cell populations present in OSCC (malignant cancer cells, $\mathrm{T}$ cells, dendritic cells, macrophages, endothelial cells and fibroblasts). Data are shown from OSCC tumors analyzed by scRNA-seq in Puram et al. [28] (GSE103322). The red dots represent the median values. The indicated $p$ values were obtained with the Kruskal-Wallis test. 


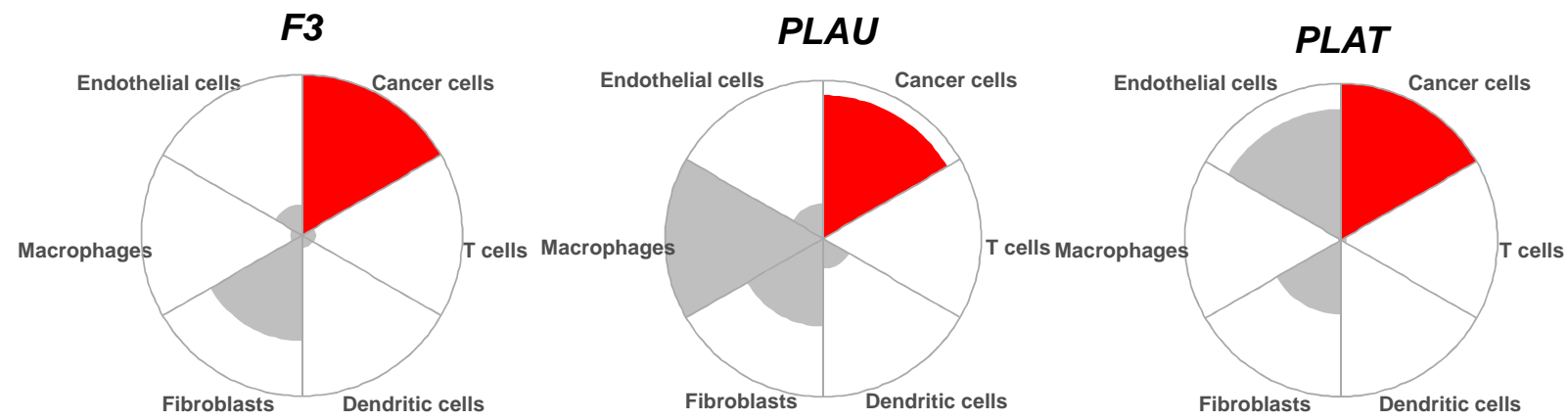

PLAUR

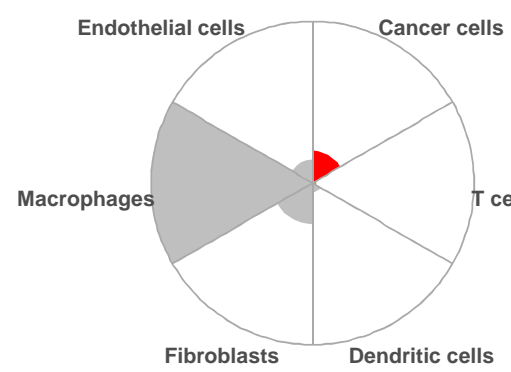

F2R

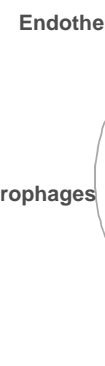

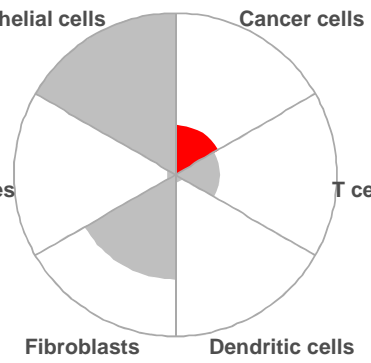

SERPINE1

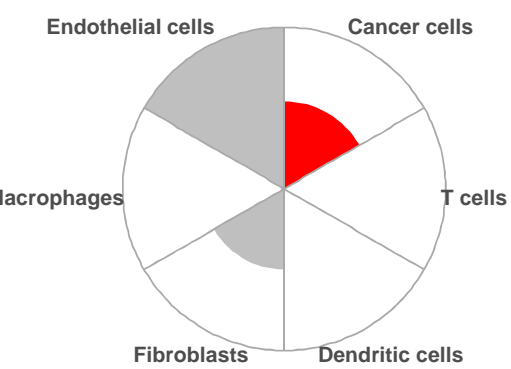

Figure 5. Polar plots showing the mRNA expression of the core coagulome genes (F3, PLAU, PLAT, PLAUR, F2R and SERPINE1) in different cell subpopulations of OSCC. The graphs show the relative expression of each gene in the different cell types (malignant cancer cells, T cells, dendritic cells, macrophages, endothelial cells and fibroblasts) using scRNA-seq data from GSE103322. In each case, the cell type with the highest expression (median) was set as maximum. Note that cancer cells are shown in red.

We further exploited scRNA-seq data to analyze the intra-tumoral heterogeneity of the coagulome in cancer cells. Firstly, the violin plot representation shown in Figure 6 confirmed the high inter-tumor heterogeneity that was observed with TCGA data: out of the ten tumors analyzed with scRNA-seq, four tumors strongly expressed $F 3$ and six expressed high levels of PLAU. In each individual tumor, great heterogeneity in F3 and PLAU mRNA expression was found among cancer cells (Figure 6). Importantly, this intra-tumor heterogeneity led to the coexistence of «opposite» transcriptional programs, i.e., procoagulant (high levels of F3) vs. profibrinolytic (high levels of $P L A U$ ) within individual tumors. We examined the extent to which F3 and PLAU expression overlapped in the cancer cells of each tumor (Figure 7). Within each OSCC, some cancer cells expressed both F3 and PLAU. However, there were also separate contingents of cancer cells that presented opposite transcriptional programs, i.e., only F3 or only PLAU (Figure 7). Depending on the tumor, the extent of the overlap in F3 and PLAU mRNA expression varied greatly, from 12 to $84 \%$ of cancer cells. We next performed a comparison of the transcriptomic profiles of cancer cells exclusively expressing F3 vs. PLAU with a GSEA analysis (Figure S4). A representative analysis, shown for example for one tumor (\#20) (selected due to the availability of the highest number of cells), indicated that the intra-tumor heterogeneity was related to the hallmark «Epithelial-Mesenchymal Transition» (Figure S4). The data supported the existence of specific biological properties of cancer cells underpinning this striking intra-tumor heterogeneity and the coexistence of procoagulant and profibrinolytic programs. 

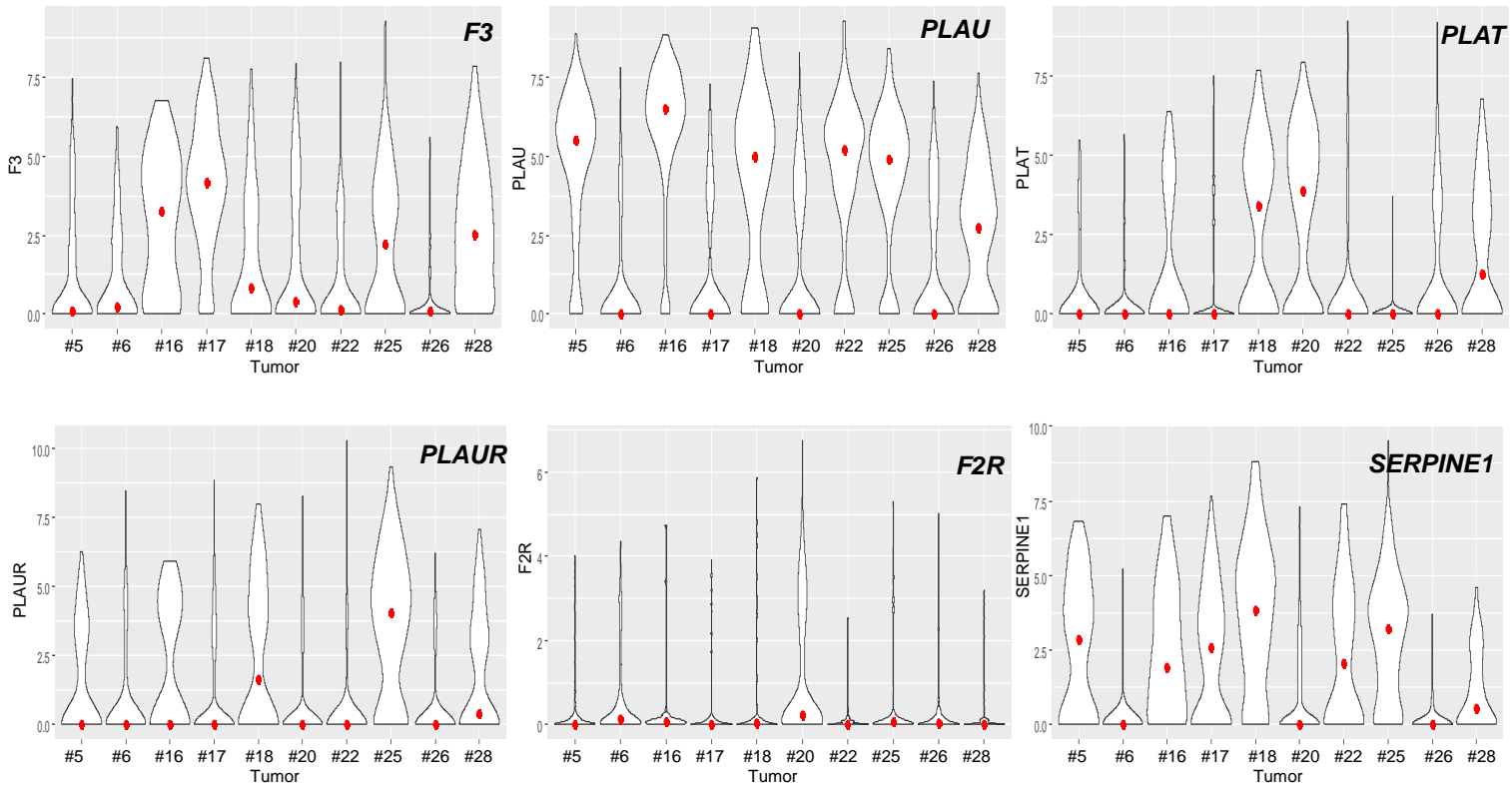

Figure 6. Intra-tumor heterogeneity of the coagulome of malignant cells from individual OSCC Violin plots showing the expression of the core coagulome genes (F3, PLAU, PLAT, PLAUR, F2R and SERPINE1) in cancer cells from different OSCC tumors analyzed in GSE103322. Each individual tumor was identified according to the study by Puram et al. [28]. The red dots represent the median value for the expression of each gene.

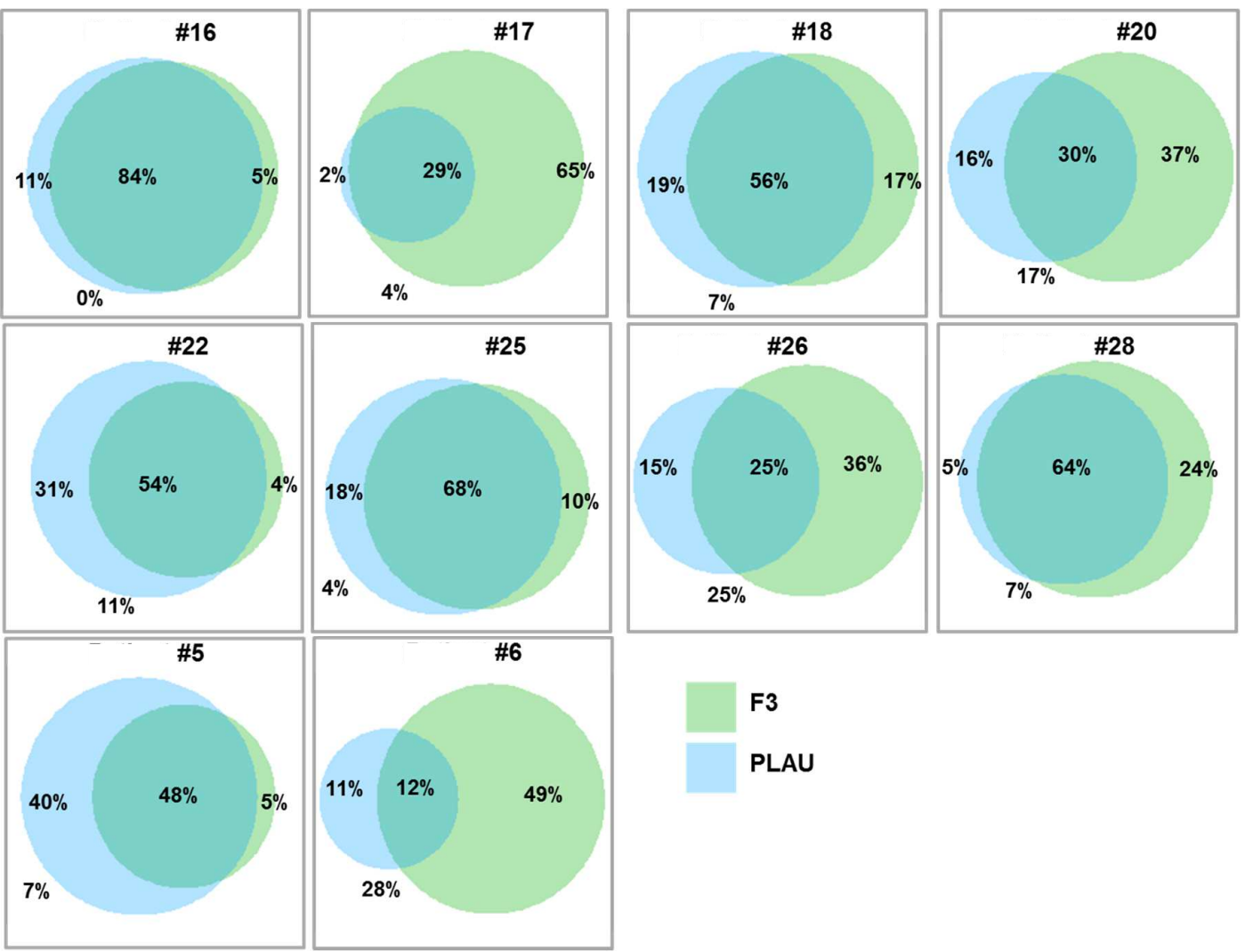

Figure 7. Overlap in F3 and PLAU expression in cancer cells from individual tumors. Venn diagrams examining the overlap between the pro-coagulant (F3-positive) and pro-fibrinolytic (PLAU-positive) transcriptional programs in cancer cells from individual tumors (GSE103322). Note that the extent of the overlap of $F 3$ and PLAU expression varied greatly depending on the tumor analyzed, ranging from $12 \%$ to $84 \%$ of cancer cells. 


\subsection{A Link between the Coagulome and the TME of OSCC}

The coagulation cascade is a potent source of inflammation in the context of acute wound healing, and it may also have a role as a regulator of the TME [24,26]. We explored the possibility that tumors from TCGA, stratified according to their expression of $F 3$, might differ in their overall cellular composition. We used the ESTIMATE algorithm to compare the content of stromal and immune cells in OSCC (Figure 8A). We found no significant differences in the stromal or immune scores between tumors with high vs. low F3 expression (Figure 8A). These negative conclusions suggested that the coagulation process does not strongly alter the cellular composition of OSCC.

\section{A}
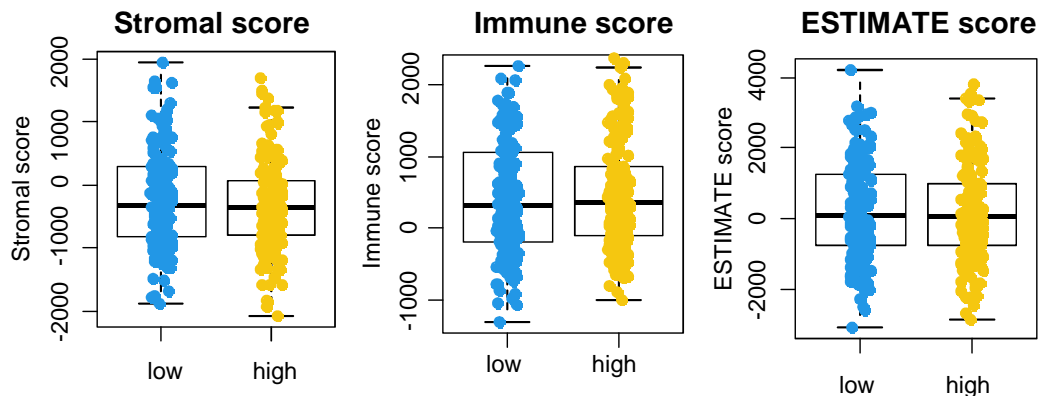

B
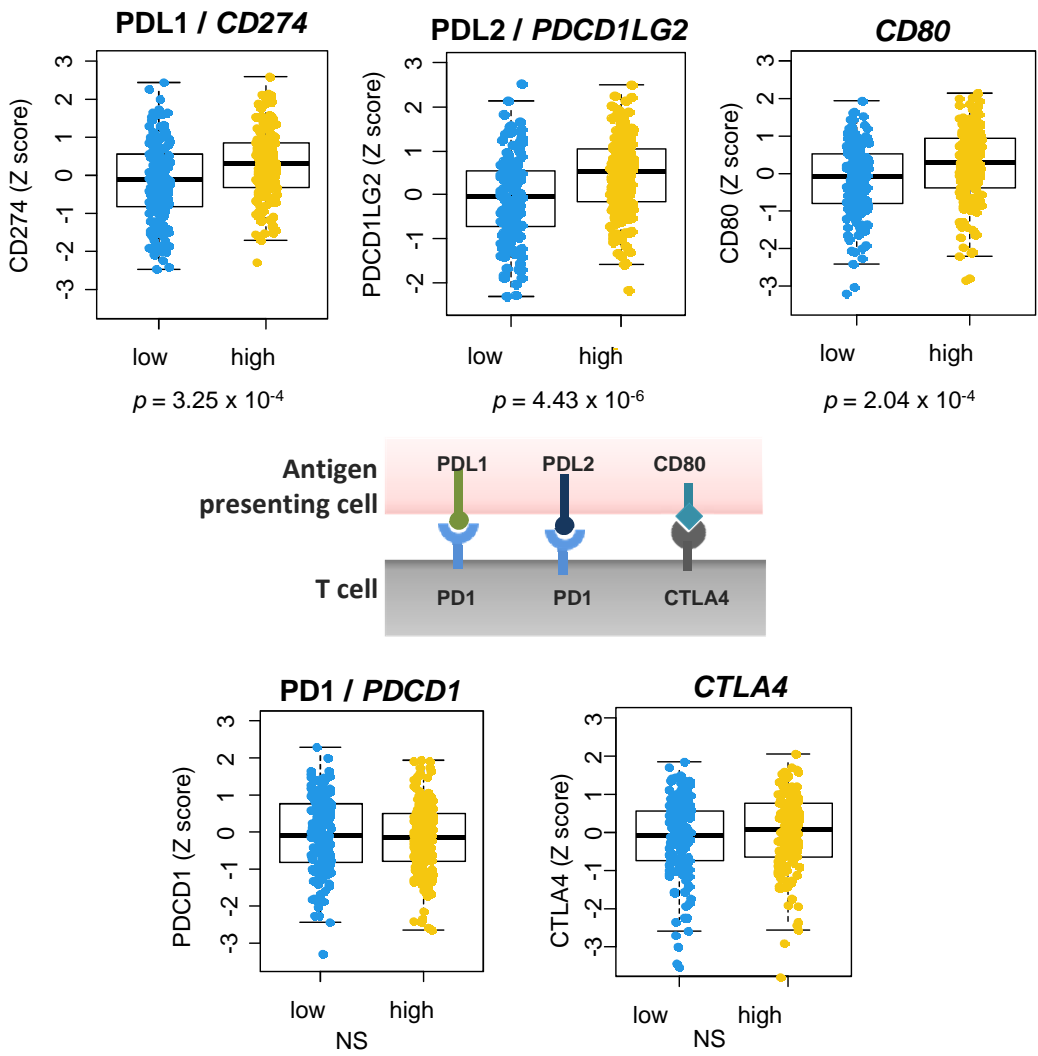

Figure 8. Stromal and immune infiltration and immune checkpoint expression in OSCC tumors. (A) Using TCGA data, the stromal and immune infiltrate was assessed in low F3 (<median, $n=158$ ) vs. high F3 (>median, $n=158$ ) OSCC tumors from TCGA using the ESTIMATE algorithm. (B) Boxplots showing the expression of immune checkpoint molecules (CD274/PD-L1, PDCD1LG2/PD-L2, CD80, PDCD1/PD1, CTLA4) in OSCC with low F3 vs. high F3 expression.

Next, we wanted to address the expression of the important immune checkpoints that control the tumor immune response and that are targeted with ICI [22]. Interestingly, an analysis using bulk RNA-seq data showed that OSCC with high F3 (i.e., above median) 
expressed higher levels of the immune checkpoints CD274/PD-L1, PDCD1LG2/PD-L2 and CD80/B7-H1 (Figure 8B). We noted a 1.60-fold $\left(p=3.26 \times 10^{-4}\right), 1.86$-fold $\left(p=4.43 \times 10^{-6}\right)$ and 1.42-fold $\left(p=2.04 \times 10^{-4}\right)$ increase in the median expression in high F3-expressing tumors, respectively. In order to validate and extend these conclusions obtained with bulk RNA-seq data from TCGA, we stratified the OSCC tumors analyzed with scRNA-seq into two groups according to $F 3$ expression in the cancer lineage (Figure 9). The violin plots shown in Figure 9 show the mRNA levels of the different checkpoints in the main cell types analyzed by Puram et al. [28]. Strikingly, tumors with a strong expression of $F 3$ in cancer cells displayed high levels of the immune checkpoints CD274/PD-L1, PDCD1LG2/PD-L2 and CD80/B7-H1 in the tumor-infiltrating dendritic cells (DC) (median levels of 4.45 in high $F 3$ vs. 0.10 in low F3; 4.01 in high $F 3$ vs. 0 in low F3 and 4.78 in high F3 vs. 0 in low F3, respectively) (Figure 9).

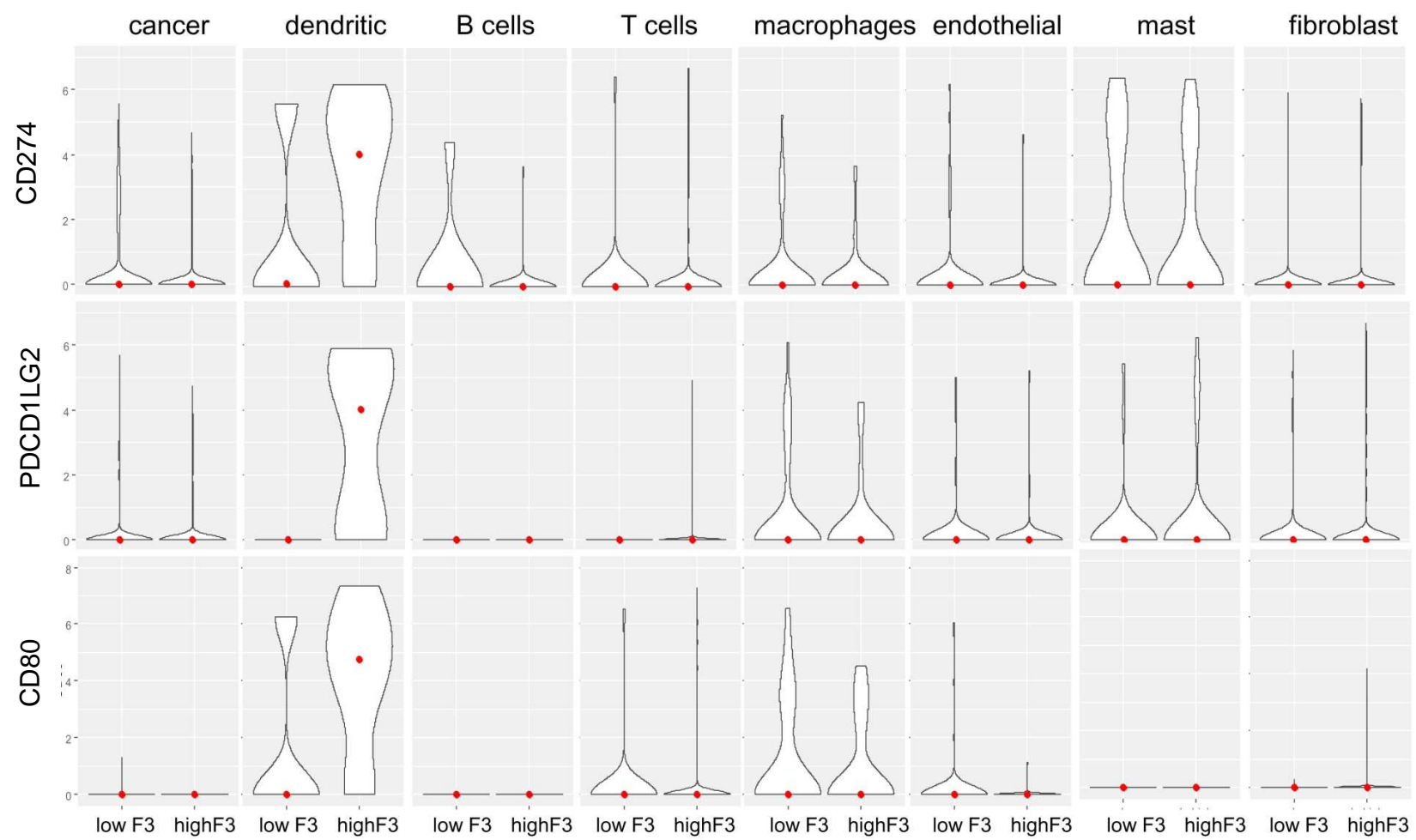

Figure 9. Single-cell analysis of the expression of immune checkpoint molecules in tumors stratified according to the expression of $F 3$ in cancer cells. Violin plots showing the expression of CD274/PD-L1, PDCD1LG2/PD-L2 and CD80 in the different cell populations from the GSE103322 cohort, including cancer cells, B cells, T cells, macrophages, endothelial cells, fibroblasts, dendritic cells, mast cells.

\subsection{The Coagulome of OSCC Cells In Vitro}

To validate our findings in vitro, we compared the coagulomes of three OSCC cell lines (PECA/PJ34, PECA/PJ41 and SCC9) to glioma cells (A172, SW1088 and U118MG) by immunoblotting (Figure 10A). High expression levels of TF and uPA were detected in the three OSCC cell lines, while tPA was only detected at faint levels. The data from genomic analyses provide a snapshot of basal tumor gene expression. We wondered whether the coagulome of OSCC might encounter variations upon exposure of OSCC to genotoxic therapeutic agents, such as the commonly used chemotherapeutics cisplatin, 5-fluorouracile and paclitaxel. We exposed OSCC cells to these chemotherapeutic agents applied at their previously determined $\mathrm{IC}_{50}$ concentrations [31]. We found no significant effect of these chemotherapeutic agents on TF/uPA expression, suggesting a relative stability of the coagulome in this context (Figure S5). 
A

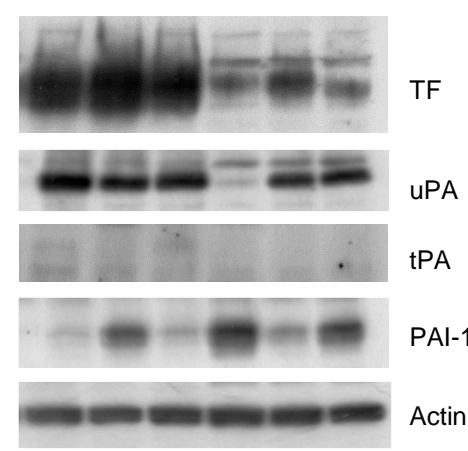

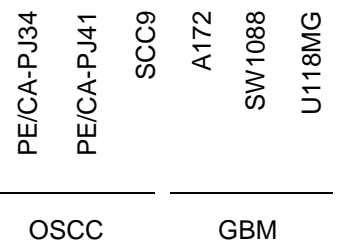

B
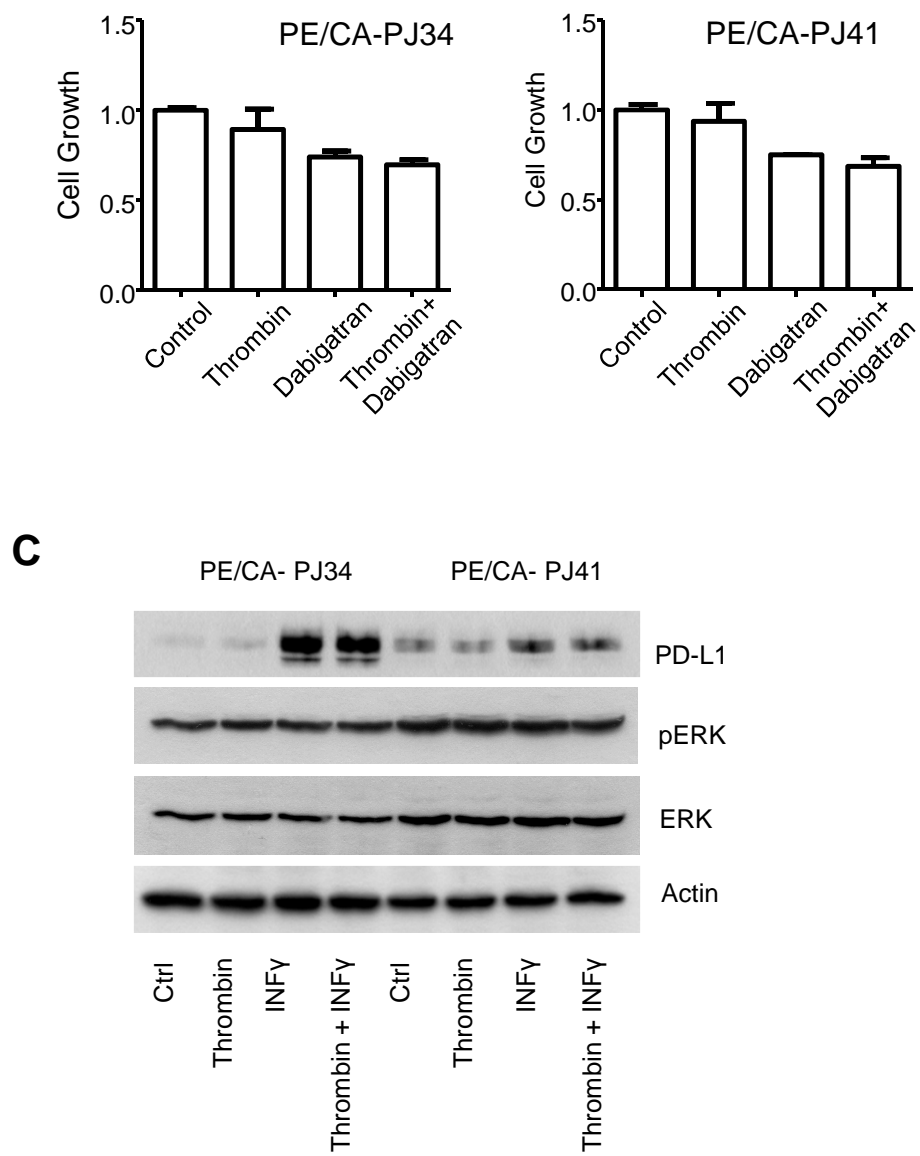

Figure 10. Immunoblot analysis of the coagulome in OSCC and the lack of a direct effect of thrombin on the growth of OSCC cells. (A) Immunoblot analysis of the expression of the Tissue Factor (F3), uPA (PLAU), tPA (PLAT) and PAI-1 (SERPINE1) in OSCC cell lines (PE/CA-PJ34, PE/CA-PJ41, SCC9) compared to GBM cell lines (A172, SW1088, U118MG). Actin was provided as a control. Protein extracts were prepared from the indicated cell lines as indicated. Note that the blots are representative of at least 3 independent experiments. (B) Lack of a direct effect of thrombin on the growth of OSCC cells in vitro. Thrombin $(10 \mathrm{NIHU} / \mathrm{L})$ and dabigatran $(1 \mu \mathrm{M})$ were maintained for $72 \mathrm{~h}$, and cancer cell growth was measured with crystal violet and normalized by taking the control condition as reference. (C) Lack of a direct effect of thrombin on the expression of the immune checkpoint molecule CD274/PD-L1 and ERK phosphorylation in OSCC cells. Thrombin (10 NIHU/L) was applied at the same time as Interferon-Gamma $(10 \mathrm{ng} / \mathrm{mL})$, used here as an inducer of PD-L1 expression. The indicated markers were analyzed after $18 \mathrm{~h}$ of incubation.

Next, we envisioned the possibility that the interaction of thrombin with its receptor PAR-1 might modulate the growth of OSCC cells in vitro. No expression of the PAR1 receptor was detected in any of the three OSCC cell lines. To examine the possibility that a small pool of PAR1 receptors expressed by OSCC cells might be functionally active, we exposed OSCC cells to thrombin $(10 \mathrm{NIHU} / \mathrm{L})$ and/or dabigatran $(1 \mu \mathrm{M})$. We found no effect of thrombin on the growth of OSCC cells in this setting (Figure 10B). Neither the expression of the immune checkpoint CD274/PD-L1 nor the phosphorylation levels of the oncogenic kinases ERK1/2 were modulated by thrombin (Figure 10C). These results suggested that OSCC cells do not directly respond to thrombin.

\section{Discussion}

Using genomic data from different sources, we examined the expression of the six genes that define the «core» coagulome, i.e., genes that contribute to coagulation and 
fibrinolysis in multiple, if not all, experimental systems [13]. We found surprisingly high expression levels of F3 (TF) and PLAU (uPA), the two key antipodal actors of coagulation/fibrinolysis, in OSCC. Among the tumor types included in TCGA, OSCC was identified as the tumor type with the highest mRNA expression levels of F3 and PLAU. Our in vitro studies confirmed the particularity of the coagulome of OSCC cells, further extending the conclusions of previous studies reporting that both TF and uPA are detectable at high protein levels in OSCC [13,32]. Overall, the coagulome of OSCC appears to be genetically stable. An inverse correlation between locus-specific DNA methylation and F3/ PLAU mRNA was observed, in agreement with previous reports that documented the regulation of F3 expression by DNA methylation in tumors other than OSCC [33]. Strikingly, we obtained data supporting the existence of great inter- and intra-tumor heterogeneity. At the single-cell level, we demonstrated that cancer cells with distinct pro-coagulant and pro-fibrinolytic transcriptional programs coexisted within each OSCC. We also found a potential link between the coagulation process and the TME of OSCC. The tumors with high expression levels of $F 3$ had higher levels of the key immune checkpoint molecules CD274/PD-L1, PDCD1LG2/PD-L2 and CD80. At the single-cell resolution, the increased expression of these immune checkpoint molecules was found to be striking in DC.

Outside of the therapeutic context, OSCC is considered to be at low risk of systemic thrombotic events $[7,8]$, a fact that has been discussed in recent literature as a paradox, considering its high expression levels of TF [8]. In the present study, we identified the high expression of PLAU (uPA), possibly counteracting clot formation via fibrinolysis, as an important element in the explanation of this paradox. Great inter-tumor heterogeneity was also observed. Our observations reinforce the notion that coagulation and fibrinolysis operate simultaneously in OSCC, in agreement with the observation that OSCC patients often have high preoperative levels of D-dimers [34]. Predicting which patients might benefit from anticoagulation in the therapeutic context remains challenging [1,2]. The finding that subpopulations of pro-coagulant and pro-fibrinolytic cancer cells coexist within individual OSCC illustrates the complex challenges posed by intra-tumor heterogeneity [35]. Based on our observations, we suggest that even OSCC with the highest global pro-coagulant transcriptomic profile might be at risk of local hemorrhage due to the presence of a fibrinolytic contingent. Our observations and those of others open up the possibility that EMT might be linked, or even contribute, to this heterogeneity [36]. More studies are required to explore the biological events that underpin the local and potentially dynamic regulation of the coagulome of OSCC.

Besides the regulation of hemostasis, the coagulation cascade potentially holds broad implications for cancer biology. Coagulation may modulate cancer growth directly or target cells of the TME $[15,19]$. The existence of a direct regulation of cancer cells via PAR1 activation by thrombin was suggested in various types of solid tumors [37-39]. Its broad relevance is, however, a matter of discussion [40]. We found no expression of PAR1 (F2R) at the protein level in OSCC cells, and in our experimental conditions, thrombin had no detectable effect on the growth of OSCC cells, their activation of the oncogenic kinases ERK1/2 or the expression of the checkpoint molecule CD274/PD-L1. Meanwhile, our analysis of single-cell RNA-seq data indicated that F2R/PAR1 is strongly expressed in endothelial cells and cancer-associated fibroblasts, but not in cancer cells in OSCC. These data suggest that coagulation and thrombin, rather than directly modulating the behavior of OSCC cells, might exert some of their effects on the TME of these tumors. Importantly, the notion that the coagulation process might influence the different cell populations that constitute the TME is mounting [41]. The possibility that the tumor coagulome and the TME mutually regulate each other deserves further study when considering the increasingly recognized role of the TME as a therapeutic target $[20,21]$.

We examined the possible impact of coagulation on the immune cells within the TME of OSCC $[20,21]$. We noted no significant correlation between the expression of F3 in OSCC and the density of the infiltrate of cells of the adaptive immune system, suggesting that coagulation does not contribute to an «immune-excluded» phenotype in 
OSCC [23]. Importantly, however, tumors expressing high levels of $F 3$ had significantly higher levels of the immune checkpoint molecules CD274/PD-L1, PDCD1LG2/PD-L2 and CD80. The interaction between PD1 and its ligands PD-L1 and PD-L2 is a key checkpoint of the adaptive immune response against tumors [22]. Its targeting with nivolumab or pembrolizumab is approved for the treatment of recurrent/metastatic stages of HNSCC, as based on evidence obtained in phase 3 trials [22]. When we examined the tumor composition at the single-cell level, we found that DC displayed marked differences in the expression of these checkpoint molecules depending on the F3 expression in the cancer cells of the tumor. This observation is important because DC are widely recognized as key antigen-presenting cells in the TME and they support anti-cancer adaptive immunity by activating T cells in the TME [42,43]. In the context of sepsis, a previous study reported PAR1 signaling on the surface of DC as a step that can amplify inflammation [44]. We did not detect F2R/PAR1 mRNA in DC in the single-cell RNA-seq analysis of OSCC, questioning the relevance of a direct control of DC by thrombin and the coagulation cascade in the TME. Our study, therefore, opens up the possibility that the coagulation cascade may indirectly regulate the expression of key immune checkpoint molecules in DC, for example by favoring a hypoxic microenvironment. The possibility that a high expression of F3/activation of coagulation might contribute to immune evasion in OSCC deserves to be experimentally tested in future studies. Importantly, recent studies are pointing to specific mechanisms through which some of the actors of the coagulation cascade may regulate tumor-specific adaptive immunity and antigen presentation [45]. Actioning the coagulome based on this rationale might be a promising strategy to boost the efficacy of the immune system, especially in cancer patients receiving immune checkpoint blockers [46].

\section{Conclusions}

OSCC express high levels of F3/TF and PLAU/uPA and these tumors constitute an interesting model for the study of coagulation in cancer. The study of the coagulome is nevertheless complex, as illustrated by the existence of great inter- and intra-tumor heterogeneity. Importantly, our findings suggest that the biological effects of the coagulome extend beyond the regulation of hemostasis. We observed that OSCC with high levels of F3 express high levels of the key immune checkpoint molecules CD274/PD-L1, PDCD1LG2/PD-L2 and CD80 in DC. Studying how the process of coagulation could be linked to the regulation of the local immune microenvironment promises to bring new insights on the regulation of the TME, with rich perspectives in terms of biomarkers and therapeutics.

Supplementary Materials: The following supporting information can be downloaded at: https: / / www.mdpi.com/article/10.3390/cancers14020460/s1, Table S1: Clinical characteristics of OSCC patients in TCGA; Table S2: Frequency of alterations/DNA seq in OSCC in TCGA; Figure S1: Expression of the coagulome and association with TNM/grade in OSCC; Figure S2: Expression of the coagulome and association with age and the smoking status in OSCC; Figure S3: Link between F3, PLAU and PLAT gene expression and OSCC patient survival; Figure S4: GSEA analysis of F3 expressing cancers cells vs. PLAU expressing cancer cells; Figure S5: Immunoblot analysis of TF, uPA and PAI-1 in OSCC cells exposed to chemotherapeutic agents.

Author Contributions: M.L. (Marine Lottin), Conceptualization, Investigation, Manuscript Reviewing; S.S., Conceptualization, Manuscript Reviewing; J.F., Investigation, Manuscript Reviewing; F.R., Investigation, Manuscript Reviewing; J.D., Manuscript Reviewing; J.B., Manuscript Reviewing; D.C., Manuscript Reviewing; M.-A.S., Manuscript Reviewing; Y.M., Manuscript Reviewing; M.L. (Michele Lamuraglia), Manuscript Reviewing; A.G., Conceptualization, Funding Acquisition, Project Administration, Investigation, Writing of the Original Draft; Z.S., Conceptualization, All Statistical Analyses, Writing of the Original Draft. All authors have read and agreed to the published version of the manuscript.

Funding: This research received no external funding.

Institutional Review Board Statement: Not applicable. 
Informed Consent Statement: Not applicable.

Data Availability Statement: The data from TCGA and GSE103322 are publicly available. The datasets used in the current study are available from the corresponding author.

Acknowledgments: We would like to thank the patients, physicians and scientists who contributed the data that were analyzed here. We are grateful to the Ligue contre le Cancer and CHU Amiens Picardie for supporting the research in our laboratory.

Conflicts of Interest: The authors declare no conflict of interest.

$\begin{array}{ll}\text { Abbreviations } \\ \text { CAF } & \text { cancer-associated fibroblasts } \\ \text { DC } & \text { dendritic cells } \\ \text { HNSCC } & \text { Head and Neck Squamous Cell Carcinoma } \\ \text { OSCC } & \text { Oral Squamous Cell Carcinoma } \\ \text { PAI-1 } & \text { plasminogen activator inhibitor-1 } \\ \text { PAR-1 } & \text { protease-activated receptor-1 } \\ \text { RSEM } & \text { RNA-Seq by Expectation-Maximization } \\ \text { TAM } & \text { tumor-associated macrophages } \\ \text { TCGA } & \text { The Cancer Genome Atlas } \\ \text { TF } & \text { Tissue Factor } \\ \text { TME } & \text { tumor microenvironment } \\ \text { uPA } & \text { urokinase-type plasminogen activator } \\ \text { uPAR } & \text { urokinase-type plasminogen activator receptor }\end{array}$

\section{References}

1. Farge, D.; Frere, C.; Connors, J.M.; Ay, C.; Khorana, A.A.; Munoz, A.; Brenner, B.; Kakkar, A.; Rafii, H.; Solymoss, S.; et al. 2019 international clinical practice guidelines for the treatment and prophylaxis of venous thromboembolism in patients with cancer. Lancet Oncol. 2019, 20, e566-e581. [CrossRef]

2. Khorana, A.A.; Cohen, A.T.; Carrier, M.; Meyer, G.; Pabinger, I.; Kavan, P.; Wells, P. Prevention of venous thromboembolism in ambulatory patients with cancer. ESMO Open 2020, 5, e000948. [CrossRef]

3. Chow, L.Q.M. Head and Neck Cancer. N. Engl. J. Med. 2020, 382, 60-72. [CrossRef] [PubMed]

4. Grover, S.P.; Hisada, Y.M.; Kasthuri, R.S.; Reeves, B.N.; Mackman, N. Cancer Therapy-Associated Thrombosis. Arterioscler. Thromb. Vasc. Biol. 2021, 41, 1291-1305. [CrossRef] [PubMed]

5. Argiris, A.; Li, S.; Savvides, P.; Ohr, J.P.; Gilbert, J.; Levine, M.A.; Chakravarti, A.; Haigentz, M.; Saba, N.F.; Ikpeazu, C.V.; et al Phase III Randomized Trial of Chemotherapy With or Without Bevacizumab in Patients with Recurrent or Metastatic Head and Neck Cancer. J. Clin. Oncol. 2019, 37, 3266-3274. [CrossRef]

6. Bergamini, C.; Ferris, R.L.; Xie, J.; Mariani, G.; Ali, M.; Holmes, W.C.; Harrington, K.; Psyrri, A.; Cavalieri, S.; Licitra, L. Bleeding complications in patients with squamous cell carcinoma of the head and neck. Head Neck 2021, 43, 2844-2858. [CrossRef]

7. Moubayed, S.P.; Eskander, A.; Mourad, M.W.; Most, S.P. Systematic review and meta-analysis of venous thromboembolism in otolaryngology-head and neck surgery. Head Neck 2017, 39, 1249-1258. [CrossRef] [PubMed]

8. Haen, P.; Mege, D.; Crescence, L.; Dignat-George, F.; Dubois, C.; Panicot-Dubois, L. Thrombosis Risk Associated with Head and Neck Cancer: A Review. Int. J. Mol. Sci. 2019, 20, 2838. [CrossRef]

9. Rak, J.; Yu, J.L.; Luyendyk, J.; Mackman, N. Oncogenes, Trousseau Syndrome, and Cancer-Related Changes in the Coagulome of Mice and Humans. Cancer Res. 2006, 66, 10643-10646. [CrossRef] [PubMed]

10. Magnus, N.; Gerges, N.; Jabado, N.; Rak, J. Coagulation-related gene expression profile in glioblastoma is defined by molecular disease subtype. J. Thromb. Haemost. 2013, 11, 1197-1200. [CrossRef]

11. Tawil, N.; Bassawon, R.; Rak, J. Oncogenes and Clotting Factors: The Emerging Role of Tumor Cell Genome and Epigenome in Cancer-Associated Thrombosis. Semin. Thromb. Hemost. 2019, 45, 373-384. [CrossRef]

12. Dunbar, A.; Bolton, K.L.; Devlin, S.M.; Sanchez-Vega, F.; Gao, J.; Mones, J.V.; Wills, J.; Kelly, D.; Farina, M.; Cordner, K.B.; et al Genomic profiling identifies somatic mutations predicting thromboembolic risk in patients with solid tumors. Blood 2021, 137, 2103-2113. [CrossRef]

13. Saidak, Z.; Soudet, S.; Lottin, M.; Salle, V.; Sevestre, M.-A.; Clatot, F.; Galmiche, A. A pan-cancer analysis of the human tumor coagulome and its link to the tumor immune microenvironment. Cancer Immunol. Immunother. 2021, 70, 923-933. [CrossRef] [PubMed]

14. Falanga, A.; Schieppati, F.; Russo, L. Pathophysiology 1. Mechanisms of Thrombosis in Cancer Patients. In Thrombosis and Hemostasis in Cancer; Soff, G., Ed.; Cancer Treatment and Research; Springer: Cham, Switzerland, 2019; Volume 179, pp. 11-36. ISBN 978-3-030-20314-6. 
15. Unruh, D.; Horbinski, C. Beyond thrombosis: The impact of tissue factor signaling in cancer. J. Hematol. Oncol. 2020, 13, 93. [CrossRef] [PubMed]

16. Pavón, M.A.; Arroyo-Solera, I.; Céspedes, M.V.; Casanova, I.; León, X.; Mangues, R. uPA/uPAR and SERPINE1 in head and neck cancer: Role in tumor resistance, metastasis, prognosis and therapy. Oncotarget 2016, 7, 57351-57366. [CrossRef] [PubMed]

17. Saidak, Z.; Galmiche, A.; Lottin, M.; Montes, L.; Soudet, S.; Sevestre, M.-A.; Clatot, F.; Mirghani, H. The coagulome of Head and Neck Squamous Cell Carcinoma. Oral Oncol. 2020, 114, 105068. [CrossRef]

18. desJardins-Park, H.E.; Mascharak, S.; Chinta, M.S.; Wan, D.C.; Longaker, M.T. The Spectrum of Scarring in Craniofacial Wound Repair. Front. Physiol. 2019, 10, 322. [CrossRef]

19. Dvorak, H.F. Tumors: Wounds That Do Not Heal-A Historical Perspective with a Focus on the Fundamental Roles of Increased Vascular Permeability and Clotting. Semin. Thromb. Hemost. 2019, 45, 576-592. [CrossRef]

20. Binnewies, M.; Roberts, E.W.; Kersten, K.; Chan, V.; Fearon, D.F.; Merad, M.; Coussens, L.M.; Gabrilovich, D.I.; Ostrand-Rosenberg, S.; Hedrick, C.C.; et al. Understanding the tumor immune microenvironment (TIME) for effective therapy. Nat. Med. 2018, 24, 541-550. [CrossRef]

21. Peltanova, B.; Raudenska, M.; Masarik, M. Effect of tumor microenvironment on pathogenesis of the head and neck squamous cell carcinoma: A systematic review. Mol. Cancer 2019, 18, 63. [CrossRef]

22. Cramer, J.D.; Burtness, B.; Le, Q.T.; Ferris, R.L. The changing therapeutic landscape of head and neck cancer. Nat. Rev. Clin. Oncol. 2019, 16, 669-683. [CrossRef]

23. Chen, D.S.; Mellman, I. Elements of cancer immunity and the cancer-immune set point. Nature 2017, 541, 321-330. [CrossRef] [PubMed]

24. Luyendyk, J.P.; Schoenecker, J.G.; Flick, M.J. The multifaceted role of fibrinogen in tissue injury and inflammation. Blood 2019, 133, 511-520. [CrossRef]

25. Fan, Q.; Ma, Q.; Bai, J.; Xu, J.; Fei, Z.; Dong, Z.; Maruyama, A.; Leong, K.W.; Liu, Z.; Wang, C. An implantable blood clot-based immune niche for enhanced cancer vaccination. Sci. Adv. 2020, 6, eabb4639. [CrossRef]

26. Cantrell, R.; Palumbo, J.S. The thrombin-inflammation axis in cancer progression. Thromb. Res. 2020, 191, S117-S122. [CrossRef]

27. Cancer Genome Atlas Network Comprehensive genomic characterization of head and neck squamous cell carcinomas. Nature 2015, 517, 576-582. [CrossRef] [PubMed]

28. Puram, S.V.; Tirosh, I.; Parikh, A.S.; Patel, A.P.; Yizhak, K.; Gillespie, S.; Rodman, C.; Luo, C.L.; Mroz, E.A.; Emerick, K.S. Single-cell transcriptomic analysis of primary and metastatic tumor ecosystems in head and neck cancer. Cell 2017, 171, 1611-1624. [CrossRef]

29. Yoshihara, K.; Shahmoradgoli, M.; Martínez, E.; Vegesna, R.; Kim, H.; Torres-Garcia, W.; Treviño, V.; Shen, H.; Laird, P.W.; Levine, D.A. Inferring tumour purity and stromal and immune cell admixture from expression data. Nat. Commun. 2013, 4, 2612. [CrossRef]

30. Subramanian, A.; Tamayo, P.; Mootha, V.K.; Mukherjee, S.; Ebert, B.L.; Gillette, M.A.; Paulovich, A.; Pomeroy, S.L.; Golub, T.R.; Lander, E.S.; et al. Gene set enrichment analysis: A knowledge-based approach for interpreting genome-wide expression profiles. Proc. Natl. Acad. Sci. USA 2005, 102, 15545-15550. [CrossRef] [PubMed]

31. Lailler, C.; Lamuraglia, M.; Racine, F.; Louandre, C.; Godin, C.; Chauffert, B.; Galmiche, A.; Saidak, Z. DNA damage responseand JAK-dependent regulation of PD-L1 expression in head and neck squamous cell carcinoma (HNSCC) cells exposed to 5-fluorouracil (5-FU). Transl. Oncol. 2021, 14, 101110. [CrossRef] [PubMed]

32. Christensen, A.; Kiss, K.; Lelkaitis, G.; Juhl, K.; Persson, M.; Charabi, B.W.; Mortensen, J.; Forman, J.L.; Sørensen, A.L.; Jensen, D.H.; et al. Urokinase-type plasminogen activator receptor (uPAR), tissue factor (TF) and epidermal growth factor receptor (EGFR): Tumor expression patterns and prognostic value in oral cancer. BMC Cancer 2017, 17, 572. [CrossRef]

33. Unruh, D.; Mirkov, S.; Wray, B.; Drumm, M.; Lamano, J.; Li, Y.D.; Haider, Q.F.; Javier, R.; McCortney, K.; Saratsis, A.; et al. Methylation-dependent Tissue Factor Suppression Contributes to the Reduced Malignancy of IDH1-mutant Gliomas. Clin. Cancer Res. 2019, 25, 747-759. [CrossRef]

34. Liang, Y.-J.; Mei, X.-Y.; Zeng, B.; Zhang, S.-E.; Yang, L.; Lao, X.-M.; Liao, G.-Q. Prognostic role of preoperative D-dimer, fibrinogen and platelet levels in patients with oral squamous cell carcinoma. BMC Cancer 2021, 21, 122. [CrossRef] [PubMed]

35. Vitale, I.; Shema, E.; Loi, S.; Galluzzi, L. Intratumoral heterogeneity in cancer progression and response to immunotherapy. Nat. Med. 2021, 27, 212-224. [CrossRef]

36. Chen, G.; Sun, J.; Xie, M.; Yu, S.; Tang, Q.; Chen, L. PLAU Promotes Cell Proliferation and Epithelial-Mesenchymal Transition in Head and Neck Squamous Cell Carcinoma. Front. Genet. 2021, 12, 651882. [CrossRef]

37. Auvergne, R.; Wu, C.; Connell, A.; Au, S.; Cornwell, A.; Osipovitch, M.; Benraiss, A.; Dangelmajer, S.; Guerrero-Cazares, H.; Quinones-Hinojosa, A.; et al. PAR1 inhibition suppresses the self-renewal and growth of A2B5-defined glioma progenitor cells and their derived gliomas in vivo. Oncogene 2016, 35, 3817-3828. [CrossRef] [PubMed]

38. Adams, G.N.; Sharma, B.K.; Rosenfeldt, L.; Frederick, M.; Flick, M.J.; Witte, D.P.; Mosnier, L.O.; Harmel-Laws, E.; Steinbrecher, K.A.; Palumbo, J.S. Protease-activated receptor-1 impedes prostate and intestinal tumor progression in mice. J. Thromb. Haemost. 2018, 16, 2258-2269. [CrossRef]

39. Tekin, C.; Shi, K.; Daalhuisen, J.B.; ten Brink, M.S.; Bijlsma, M.F.; Spek, C.A. PAR1 signaling on tumor cells limits tumor growth by maintaining a mesenchymal phenotype in pancreatic cancer. Oncotarget 2018, 9, 32010-32023. [CrossRef] [PubMed] 
40. Najidh, S.; Versteeg, H.H.; Buijs, J.T. A systematic review on the effects of direct oral anticoagulants on cancer growth and metastasis in animal models. Thromb. Res. 2020, 187, 18-27. [CrossRef]

41. Galmiche, A.; Rak, J.; Roumenina, L.T.; Saidak, Z. Coagulome and the tumor microenvironment: An actionable interplay. Trends Cancer 2022. in press. [CrossRef] [PubMed]

42. Murphy, T.L.; Murphy, K.M. Dendritic cells in cancer immunology. Cell Mol. Immunol. 2021, 19, 3-13. [CrossRef] [PubMed]

43. Noubade, R.; Majri-Morrison, S.; Tarbell, K.V. Beyond cDC1: Emerging Roles of DC Crosstalk in Cancer Immunity. Front. Immunol. 2019, 10, 1014. [CrossRef] [PubMed]

44. Niessen, F.; Schaffner, F.; Furlan-Freguia, C.; Pawlinski, R.; Bhattacharjee, G.; Chun, J.; Derian, C.K.; Andrade-Gordon, P.; Rosen, H.; Ruf, W. Dendritic cell PAR1-S1P3 signalling couples coagulation and inflammation. Nature 2008, 452, 654-658. [CrossRef] [PubMed]

45. Graf, C.; Wilgenbus, P.; Pagel, S.; Pott, J.; Marini, F.; Reyda, S.; Kitano, M.; Macher-Göppinger, S.; Weiler, H.; Ruf, W. Myeloid cell-synthesized coagulation factor X dampens antitumor immunity. Sci. Immunol. 2019, 4, eaaw8405. [CrossRef] [PubMed]

46. Haist, M.; Stege, H.; Pemler, S.; Heinz, J.; Fleischer, M.I.; Graf, C.; Ruf, W.; Loquai, C.; Grabbe, S. Anticoagulation with Factor Xa Inhibitors Is Associated with Improved Overall Response and Progression-Free Survival in Patients with Metastatic Malignant Melanoma Receiving Immune Checkpoint Inhibitors-A Retrospective, Real-World Cohort Study. Cancers 2021, 13, 5103. [CrossRef] 